\title{
Polarimetric nonregularity of evanescent waves
}

\author{
Andreas Norrman, ${ }^{1, *}$ (1) José J. Gil, ${ }^{2}$ Ari T. Friberg, ${ }^{3}$ and Tero Setälä ${ }^{3}$ \\ ${ }^{1}$ Max Planck Institute for the Science of Light, Staudtstraße 2, D-91058 Erlangen, Germany \\ ${ }^{2}$ Department of Applied Physics, University of Zaragoza, Pedro Cerbuna 12, 50009 Zaragoza, Spain \\ ${ }^{3}$ Institute of Photonics, University of Eastern Finland, P.O. Box 111, Fl-80101 Joensuu, Finland \\ *Corresponding author: andreas.norrman@mpl.mpg.de
}

Received 12 September 2018; accepted 1 November 2018; posted 15 November 2018 (Doc. ID 345684); published 2 January 2019

Three-dimensional polarization states of random light can be classified into regular and nonregular according to the structure of the related $3 \times 3$ polarization matrix. Here we show that any purely evanescent wave excited in total internal reflection of a partially polarized plane-wave field is always in a nonregular polarization state. The degree of nonregularity of such evanescent waves is also studied in terms of a recently advanced measure. Nonregular evanescent waves uncover new aspects of the polarimetric structure and dimensional character of electromagnetic near fields, with potential applications in nanoscale surface optics. (๑) 2019 Optical Society of America

https://doi.org/10.1364/OL.44.000215

The rapid progress in nano-optics [1] and ever-growing interest toward complex structured light [2] have witnessed the need for a full three-dimensional (3D) treatment of polarization [3-10]. In particular, whereas for two-dimensional (2D) light, such as directional beams, the polarization ellipse is restricted to a plane, evanescent waves [11,12], optical surface fields [13,14], and tightly focused light [15-17] encompass 3D polarization states with the electric field fluctuating in three orthogonal directions. The polarization states of such genuine $3 \mathrm{D}$ light fields can be classified into regular and nonregular states according to the nature of their characteristic decomposition [18]. A regular state is an incoherent composition of a pure state, a $2 \mathrm{D}$ unpolarized state, and a 3D unpolarized state, while for a nonregular state the intermediate component corresponds to a specific $3 \mathrm{D}$ state: an equiprobable mixture of two mutually orthogonal pure states with the electric-field ellipses lying in different planes in space. Nonregular polarization states constitute a significant and rich family of 3D light fields with potential applications in near-field optics and high-numerical-aperture imaging systems [19].

In this Letter, we examine the spectral polarimetric structure of an evanescent field generated by a partially polarized plane wave in total internal reflection at a lossless dielectric interface. We especially establish that any stationary, partially polarized evanescent wave is in a nonregular polarization state and thus cannot be addressed by the standard $2 \mathrm{D}$ polarization formalism. Although our focus is on evanescent waves, the analysis also reveals that any partially polarized refracted wave is in a regular polarization state. We investigate further the nonregularity of $3 \mathrm{D}$ evanescent waves by utilizing a recently introduced measure, the degree of nonregularity [19], and show that, under certain circumstances, an evanescent wave is virtually in a so-called perfect nonregular state of maximal polarimetric nonregularity. Evanescent waves endowed with nonregular 3D polarization states could be instrumental in surface light-matter interactions and optical manipulations at subwavelength scales.

The polarization characteristics of a random stationary light field are in the space-frequency domain completely described by the $3 \times 3$ spectral polarization matrix $[3,4,20]$

$$
\boldsymbol{\Phi}(\mathbf{r}, \omega)=\left\langle\mathbf{E}^{*}(\mathbf{r}, \omega) \mathbf{E}^{\mathrm{T}}(\mathbf{r}, \omega)\right\rangle .
$$

The three-component column vector $\mathbf{E}(\mathbf{r}, \omega)$ is a monochromatic realization representing the electric field at point $\mathbf{r}$ and (angular) frequency $\omega$, while the angle brackets, asterisk, and superscript $\mathrm{T}$ denote ensemble averaging, complex conjugation, and matrix transpose, respectively. The polarization matrix can always be expressed via the characteristic decomposition $[3,4]$

$$
\boldsymbol{\Phi}(\mathbf{r}, \omega)=I\left[P_{1} \hat{\boldsymbol{\Phi}}_{p}+\left(P_{2}-P_{1}\right) \hat{\boldsymbol{\Phi}}_{m}+\left(1-P_{2}\right) \hat{\boldsymbol{\Phi}}_{u}\right],
$$

where $I=\lambda_{1}+\lambda_{2}+\lambda_{3}$ is the intensity, with $\lambda_{1} \geq \lambda_{2} \geq \lambda_{3} \geq 0$ being the eigenvalues of $\boldsymbol{\Phi}(\mathbf{r}, \omega)$, while

$$
P_{1}=\hat{\lambda}_{1}-\hat{\lambda}_{2}, \quad P_{2}=1-3 \hat{\lambda}_{3}, \quad\left(0 \leq P_{1} \leq P_{2} \leq 1\right)
$$
are the indices of polarimetric purity [21-23], with $\hat{\lambda}_{j}=\lambda_{j} / I$ for $j \in\{1,2,3\}$. Furthermore,

$$
\begin{gathered}
\hat{\boldsymbol{\Phi}}_{p}=\mathbf{U} \operatorname{diag}(1,0,0) \mathbf{U}^{\dagger}, \quad \hat{\boldsymbol{\Phi}}_{m}=\frac{1}{2} \mathbf{U} \operatorname{diag}(1,1,0) \mathbf{U}^{\dagger}, \\
\hat{\boldsymbol{\Phi}}_{u}=\frac{1}{3} \mathbf{U} \operatorname{diag}(1,1,1) \mathbf{U}^{\dagger}=\frac{1}{3} \mathbf{I},
\end{gathered}
$$

where $\mathbf{U}$ is the unitary matrix that diagonalizes $\boldsymbol{\Phi}(\mathbf{r}, \omega)$, I is the $3 \times 3$ identity matrix, and the dagger stands for conjugate transpose. While the matrices $\hat{\boldsymbol{\Phi}}_{p}$ and $\hat{\boldsymbol{\Phi}}_{u}$ describe, respectively, a fully polarized state and a completely unpolarized $3 \mathrm{D}$ state, the physical interpretation of $\hat{\boldsymbol{\Phi}}_{m}$ is more involved and motivates the introduction of the notions of regular and nonregular states of polarization [18].

When $\hat{\boldsymbol{\Phi}}_{m}$ is a real matrix, it represents unpolarized 2D light, i.e., light whose electric field evolves fully randomly in 
a fixed plane. In this case (and in the special case $P_{1}=P_{2}$ for which $\hat{\boldsymbol{\Phi}}_{m}$ is absent in the characteristic decomposition), the polarization state $\boldsymbol{\Phi}(\mathbf{r}, \omega)$ is called regular, only met when the smallest eigenvalue $0 \leq \hat{m}_{3} \leq 1 / 4$ of $\operatorname{Re}\left(\hat{\boldsymbol{\Phi}}_{m}\right)$ is zero [18]. If $\hat{\boldsymbol{\Phi}}_{m}$ is instead a complex matrix, it corresponds to an equiprobable mixture of two mutually orthogonal states whose electric-field ellipses lie in different planes [18], whereby $\hat{\boldsymbol{\Phi}}_{m}$ does not represent unpolarized $2 \mathrm{D}$ light but genuine $3 \mathrm{D}$ light. The polarization state $\boldsymbol{\Phi}(\mathbf{r}, \omega)$ of such a light field, encountered when $0<\hat{m}_{3} \leq 1 / 4$, is said to be nonregular. The maximum value $\hat{m}_{3}=1 / 4$ is saturated when the middle component $\hat{\boldsymbol{\Phi}}_{m}$ is in a perfect nonregular state, namely, a state which is an equiprobable mixture of a circularly polarized state and a mutually orthogonal linearly polarized state [19]. The nonregularity of the full state $\boldsymbol{\Phi}(\mathbf{r}, \omega)$ can be characterized quantitatively by means of the degree of nonregularity [19]

$$
P_{N}=4\left(P_{2}-P_{1}\right) \hat{m}_{3}
$$

with the minimum $P_{N}=0$ always, and only, taking place for regular states, while values in the range $0<P_{N} \leq 1$ are signatures of nonregularity. The maximum $P_{N}=1$ is reached merely for maximally nonregular states with $P_{1}=0, P_{2}=1$, and $\hat{m}_{3}=1 / 4$. Thus, $P_{N}=1$ corresponds exclusively to $3 \mathrm{D}$ polarization states satisfying $\boldsymbol{\Phi}(\mathbf{r}, \omega)=I \hat{\boldsymbol{\Phi}}_{m}$ and $\hat{m}_{3}=1 / 4$, implying that all maximally nonregular states are perfect nonregular states. an $s$-polarized and a $p$-polarized part, hits the boundary at the angle of incidence $\theta$. The electric field of the transmitted wave in medium 2 then reads, in Cartesian coordinates, as $[9,11,12]$

$$
\mathbf{E}(\mathbf{r}, \omega)=\frac{1}{\chi}\left(\begin{array}{c}
-\gamma t_{p} E_{p} \\
\chi t_{s} E_{s} \\
\sin \theta t_{p} E_{p}
\end{array}\right) \mathrm{e}^{\mathrm{i} k_{1}(\sin \theta x+\gamma z)},
$$

where $E_{s}$ and $E_{p}$ are, respectively, the ( $\omega$-dependent) complex field amplitudes of the $s$ - and $p$-polarized components of the incident beam in medium 1 . Moreover, the ( $\omega$-dependent) quantities $\chi$ and $\gamma$ are defined as

$$
\chi=\sqrt{\sin ^{2} \theta+|\gamma|^{2}}, \quad \gamma=\frac{1}{\tilde{n}} \sqrt{1-(\tilde{n} \sin \theta)^{2}},
$$

with the ratio $\tilde{n}=n_{1} / n_{2}$, the Fresnel transmission coefficients $t_{s}$ and $t_{p}$ for the two polarizations are given by

$$
t_{s}=\frac{2 \cos \theta}{\cos \theta+\gamma}, \quad t_{p}=\frac{2 \tilde{n}^{2} \cos \theta \chi}{\cos \theta+\tilde{n}^{2} \gamma},
$$

and $k_{1}$ is the ( $\omega$-dependent) wave number in medium 1 . For $\tilde{n}>1$ and $\theta>\theta_{\mathrm{c}}$, where $\theta_{\mathrm{c}}=\arcsin \tilde{n}^{-1}$ is the critical angle, $\gamma$ is imaginary, amounting to an evanescent wave. Otherwise $\gamma$ is real, corresponding to a refracted wave.

The spectral polarization matrix for the transmitted field is now obtained from Eqs. (1) and (6), viz.,

$$
\boldsymbol{\Phi}(\mathbf{r}, \omega)=\frac{1}{\chi^{2}}\left(\begin{array}{ccc}
|\gamma|^{2} w_{p} & -\gamma^{*} \chi \sqrt{w_{s} w_{p}}|\mu| \mathrm{e}^{-\mathrm{i} \varphi} & -\gamma^{*} \sin \theta w_{p} \\
-\gamma \chi \sqrt{w_{s} w_{p}}|\mu| \mathrm{e}^{\mathrm{i} \varphi} & \chi^{2} w_{s} & \sin \theta \chi \sqrt{w_{s} w_{p}}|\mu| \mathrm{e}^{\mathrm{i} \varphi} \\
-\gamma \sin \theta w_{p} & \sin \theta \chi \sqrt{w_{s} w_{p}}|\mu| \mathrm{e}^{-\mathrm{i} \varphi} & \sin ^{2} \theta w_{p}
\end{array}\right),
$$

Let us next consider a stationary light beam, represented as a monochromatic realization of a homogeneous plane wave, incident onto a planar interface $(z=0)$ between two uniform dielectric media (Fig. 1). Both medium $1(z<0)$ and medium $2(z>0)$, having $\left(\omega\right.$-dependent) refractive indices $n_{1}$ and $n_{2}$, respectively, are lossless, and the $x z$ plane is chosen to coincide with the plane of incidence. The incoming wave, carrying both

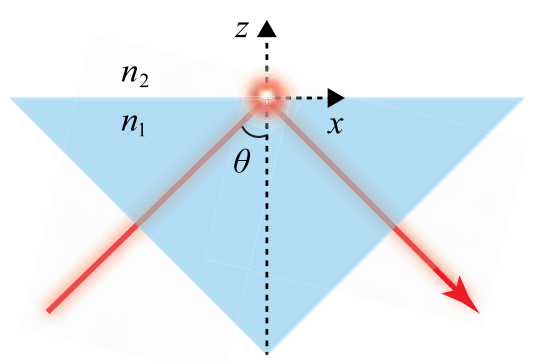

Fig. 1. Generation of a nonregular 3D evanescent wave via total internal reflection at a planar interface $(z=0)$ between two lossless dielectric media with refractive indices $n_{1}(z<0)$ and $n_{2}(z>0)$. The stationary and partially polarized incident beam impinges the surface at the angle of incidence $\theta$. where $w_{\nu}=\left|t_{\nu}\right|^{2}\left\langle\left|E_{\nu}\right|^{2}\right\rangle \mathrm{e}^{-2 k_{1} \gamma^{\prime \prime} z}$ is proportional to the energy density of the $\nu \in\{s, p\}$ polarized part of the transmitted wave at height $z$, with the double prime denoting the imaginary part. Furthermore, $\mu=\left\langle E_{s}^{*} E_{p}\right\rangle /\left(\left\langle\left|E_{s}\right|^{2}\right\rangle\left\langle\left|E_{p}\right|^{2}\right\rangle\right)^{1 / 2}$ is the correlation coefficient between the $s$ - and $p$-polarized constituents of the incident light, while $\varphi=\arg (\mu)-\arg \left(t_{s}\right)+\arg \left(t_{p}\right)$ is a phase. When $\gamma^{\prime \prime}=0$ (refracted wave), $\boldsymbol{\Phi}(\mathbf{r}, \omega)$ is position independent. We recall that the polarization-state class is determined by the middle component $\hat{\boldsymbol{\Phi}}_{m}$ in the characteristic decomposition (2), which in turn is specified by the diagonalizing unitary matrix $\mathbf{U}$. Thus, to ascertain under which circumstances the transmitted wave is regular/nonregular, we search for the eigenvalues and eigenvectors of $\boldsymbol{\Phi}(\mathbf{r}, \omega)$ in Eq. (9) and construct the respective unitary matrix.

The eigenvalues are found from the characteristic equation

$$
|\boldsymbol{\Phi}(\mathbf{r}, \omega)-\lambda \mathbf{I}|=0,
$$

which together with Eq. (9) results in

$$
\begin{gathered}
\lambda_{1}=\frac{1}{2}\left(w_{s}+w_{p}\right)\left(1+P_{1}\right), \quad \lambda_{2}=\frac{1}{2}\left(w_{s}+w_{p}\right)\left(1-P_{1}\right), \\
\lambda_{3}=0,
\end{gathered}
$$

where the polarimetric purity index $P_{1}$ explicitly reads 


$$
P_{1}=\sqrt{1-4 \frac{\left(1-|\mu|^{2}\right) w_{s} w_{p}}{\left(w_{s}+w_{p}\right)^{2}} .}
$$

The polarimetric purity index in Eq. (12) is closely related to the electromagnetic degree of coherence of an evanescent wave [11]. We especially notice that $\lambda_{3}=0$ and thus $P_{2}=1$, regardless of whether the transmitted wave is evanescent or refracted, owing to incident plane-wave illumination [24], so the last component in the characteristic decomposition (2) automatically vanishes. In addition, since a fully polarized incident beam with $\left\langle\left|E_{s}\right|^{2}\right\rangle=0$ (leading to $w_{s}=0$ ), $\left\langle\left|E_{p}\right|^{2}\right\rangle=0$ (leading to $w_{p}=0$ ), or $|\mu|=1$ results in a fully polarized transmitted wave $\left(P_{1}=1\right.$ and $\left.\lambda_{2}=0\right)$ for which the middle term in Eq. (2) also vanishes, we henceforth assume that $\left\langle\left|E_{s}\right|^{2}\right\rangle>0,\left\langle\left|E_{p}\right|^{2}\right\rangle>0$, and $|\mu|<1$. These requirements, together with $\tilde{n}>1$ and $\theta>\theta_{\mathrm{c}}$, lead to a partially polarized, genuine $3 \mathrm{D}$ evanescent wave [9].

We next determine the three (orthonormal) eigenvectors $\hat{\mathbf{u}}_{1}$, $\hat{\mathbf{u}}_{2}$, and $\hat{\mathbf{u}}_{3}$ from the eigenequation

$$
\boldsymbol{\Phi}(\mathbf{r}, \omega) \hat{\mathbf{u}}_{j}=\lambda_{j} \hat{\mathbf{u}}_{j}, \quad j \in\{1,2,3\} .
$$

For $\lambda_{3}=0$ we obtain from Eqs. (9) and (13)

$$
\hat{\mathbf{u}}_{3}=\frac{1}{\chi}\left(\begin{array}{c}
\sin \theta \\
0 \\
\gamma
\end{array}\right),
$$

with the normalization $\left|\hat{\mathbf{u}}_{3}\right|=1$, and observe that $\hat{\mathbf{u}}_{3}$ is exactly the unit-modulus wave vector of the transmitted wave $[9,11,12]$. In the case of $\lambda_{1}$ and $\lambda_{2}$, given by Eq. (11), we first define the real-valued, positive, and dimensionless quantities

$$
l_{1}=\frac{1}{2}(1+\tilde{w})\left(1+P_{1}\right), \quad l_{2}=\frac{1}{2}(1+\tilde{w})\left(1-P_{1}\right),
$$

with the ratio $\tilde{w}=w_{s} / w_{p}$. Then, on utilizing the normalizations $\left|\hat{\mathbf{u}}_{1}\right|=\left|\hat{\mathbf{u}}_{2}\right|=1$ and the relation $\tilde{w}|\mu|^{2}=\left(l_{1}-1\right)\left(1-l_{2}\right)$, Eqs. (9), (13), and (15) yield after straightforward algebra

$$
\hat{\mathbf{u}}_{1}=\frac{c}{\chi}\left(\begin{array}{c}
-\gamma^{*} \sqrt{1-l_{2}} \\
\chi \mathrm{e}^{\mathrm{i} \varphi \sqrt{l_{1}-1}} \\
\sin \theta \sqrt{1-l_{2}}
\end{array}\right), \quad \hat{\mathbf{u}}_{2}=\frac{c}{\chi}\left(\begin{array}{c}
\gamma^{*} \sqrt{l_{1}-1} \\
\chi \mathrm{e}^{\mathrm{i} \varphi \sqrt{1-l_{2}}} \\
-\sin \theta \sqrt{l_{1}-1}
\end{array}\right),
$$

where $c=1 /\left(l_{1}-l_{2}\right)^{1 / 2}$.

From the orthonormal eigenvectors $\hat{\mathbf{u}}_{1}, \hat{\mathbf{u}}_{2}$, and $\hat{\mathbf{u}}_{3}$ in Eqs. (14) and (16) we now construct the unitary matrix

$$
\mathbf{U}=\frac{1}{\chi}\left(\begin{array}{ccc}
-c \gamma^{*} \sqrt{1-l_{2}} & c \gamma^{*} \sqrt{l_{1}-1} & \sin \theta \\
c \chi \mathrm{e}^{\mathrm{i} \varphi} \sqrt{l_{1}-1} & c \chi \mathrm{e}^{\mathrm{i} \varphi} \sqrt{1-l_{2}} & 0 \\
c \sin \theta \sqrt{1-l_{2}} & -c \sin \theta \sqrt{l_{1}-1} & \gamma
\end{array}\right),
$$

and on combining Eqs. (4) and (17) we eventually end up with

$$
\hat{\boldsymbol{\Phi}}_{m}=\frac{1}{2 \chi^{2}}\left(\begin{array}{ccc}
|\gamma|^{2} & 0 & -\gamma^{*} \sin \theta \\
0 & \chi^{2} & 0 \\
-\gamma \sin \theta & 0 & \sin ^{2} \theta
\end{array}\right) .
$$

We recall that $\gamma$ is imaginary (real) for an evanescent (refracted) wave, in which case $\hat{\boldsymbol{\Phi}}_{m}$ above is complex (real) and corresponds to a nonregular (regular) polarization state. This discovery is the main result of this Letter.
One observes that the matrix $\hat{\boldsymbol{\Phi}}_{m}$ in Eq. (18) of the transmitted wave is independent of the polarization state of the incident field. Nonetheless, the weight of $\hat{\boldsymbol{\Phi}}_{m}$ in the corresponding characteristic decomposition, namely, $\boldsymbol{\Phi}(\mathbf{r}, \omega)=$ $I\left[P_{1} \hat{\boldsymbol{\Phi}}_{p}+\left(1-P_{1}\right) \hat{\boldsymbol{\Phi}}_{m}\right]$, does depend on the polarization of the excitation light via the polarimetric purity index $P_{1}$ given by Eq. (12). This property is similar in spirit to the case with propagating 2D beam fields [3]. We remark that since also the first term $\hat{\boldsymbol{\Phi}}_{p}$ is fully specified by the unitary matrix $\mathbf{U}$ in Eq. (17), the whole characteristic decomposition of $\boldsymbol{\Phi}(\mathbf{r}, \omega)$ in Eq. (9) for the transmitted wave can be explicitly determined.

We further find that $\hat{\boldsymbol{\Phi}}_{m}$ in Eq. (18) can be decomposed into an $s$-polarized part and a $p$-polarized part, i.e.,

$$
\hat{\boldsymbol{\Phi}}_{m}=\frac{1}{2}\left(\hat{\mathbf{s}}^{*} \hat{\mathbf{s}}^{\mathrm{T}}+\hat{\mathbf{p}}^{*} \hat{\mathbf{p}}^{\mathrm{T}}\right),
$$

by employing the orthonormal polarization vectors $[11,12]$

$$
\hat{\mathbf{s}}=\left(\begin{array}{l}
0 \\
1 \\
0
\end{array}\right), \quad \hat{\mathbf{p}}=\frac{1}{\chi}\left(\begin{array}{c}
-\gamma \\
0 \\
\sin \theta
\end{array}\right) .
$$

Therefore, when the transmitted wave is evanescent (refracted), the matrix $\hat{\boldsymbol{\Phi}}_{m}$ in Eq. (18) can be interpreted as an equiprobable mixture of a linear $s$-polarized state in the $y$ direction and an elliptical (linear) $p$-polarized state in the $x z$ plane.

Motivated by the above finding, we further investigate how nonregular an evanescent wave can be, and for this we employ the degree of nonregularity $P_{N}$ in Eq. (5). Because the smallest eigenvalue of $\operatorname{Re}\left(\hat{\boldsymbol{\Phi}}_{m}\right)$ in Eq. (18) is $\hat{m}_{3}=(1 / 2)(|\gamma| / \chi)^{2}$, and since $P_{2}=1$ according to Eqs. (3) and (11), one finds that

$$
P_{N}=2\left(1-P_{1}\right) \frac{|\gamma|^{2}}{\chi^{2}} \text {. }
$$

From Eqs. (7), (12), and (21) we then obtain that the fundamental upper limit for $P_{N}$ that an evanescent wave can attain is

$$
\max \left(P_{N}\right)=1-\frac{1}{2(\tilde{n} \sin \theta)^{2}-1},
$$

which is reached when the incident light possesses the properties

$$
|\mu|=0, \quad \frac{\left\langle\left|E_{s}\right|^{2}\right\rangle}{\left\langle\left|E_{p}\right|^{2}\right\rangle}=\frac{\left|t_{p}\right|^{2}}{\left|t_{s}\right|^{2}} .
$$

In this case, $P_{1}=0$, whereupon the full polarization matrix in Eq. (9) is directly proportional to the middle term in Eq. (18). Figure 2 shows the $\theta$-dependent behavior of $\max \left(P_{N}\right)$ in Eq. (22) for different values of $\tilde{n}$. We observe that when $\theta$ and $\tilde{n}$ become large, then $\max \left(P_{N}\right)$ of the evanescent wave also becomes large. For a high refractive index contrast boundary, such as GaP and air with $\tilde{n} \approx 4$ in the optical regime [25], Eq. (22) shows that the degree of nonregularity may be as high as $P_{N} \approx 0.97$, virtually corresponding to a perfect nonregular state. In such a scenario, the evanescent wave is in an equiprobable mixture of a linear $s$-polarized state and an almost circular $p$-polarized state. For a typical $\mathrm{SiO}_{2}$-air interface, on the other hand, the maximum is around $P_{N} \approx 0.71$.

In summary, we have explored the spectral polarization of random 3D evanescent waves at a lossless dielectric boundary. In particular, we showed that any partially polarized evanescent wave excited by a stationary and homogeneous plane-wave field via total internal reflection is in a nonregular polarization state. 


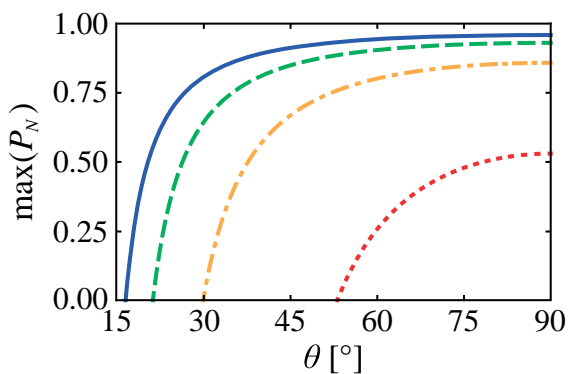

Fig. 2. Maximum of the degree of nonregularity $\max \left(P_{N}\right)$ for a partially polarized evanescent wave as a function of the angle of incidence $\theta$ for selected refractive index ratios: $\tilde{n}=3.50$ (solid blue curve), $\tilde{n}=$ 2.75 (dashed green curve), $\tilde{n}=2.00$ (dashed-dotted orange curve), and $\tilde{n}=1.25$ (dotted red curve).

The analysis also revealed that any partially polarized refracted wave is in a regular polarization state. In addition, by employing a recently established measure, the degree of nonregularity, we demonstrated that for a sufficiently high refractive index contrast surface and for a large incidence angle of the excitation beam the evanescent wave is effectively in a perfect nonregular state. Such unconventional, nonregular 3D evanescent waves provide novel insights into the polarimetric structure of electromagnetic near fields and could be exploited in surface nanophotonics.

Funding. Swedish Cultural Foundation in Finland; Academy of Finland (310511, 308393); Joensuu University Foundation; University of Eastern Finland (930350, 931726).

\section{REFERENCES}

1. L. Novotny and B. Hecht, Principles of Nano-Optics, 2nd ed. (Cambridge University, 2012).

2. H. Rubinsztein-Dunlop, A. Forbes, M. V. Berry, M. R. Dennis, D. L. Andrews, M. Mansuripur, C. Denz, C. Alpmann, P. Banzer, T. Bauer, E. Karimi, L. Marrucci, M. Padgett, M. Ritsch-Marte,
N. M. Litchinitser, N. P. Bigelow, C. Rosales-Guzmán, A. Belmonte, J. P. Torres, T. W. Neely, M. Baker, R. Gordon, A. B. Stilgoe, J. Romero, A. G. White, R. Fickler, A. E. Willner, G. Xie, B. McMorran, and A. M. Weiner, J. Opt. 19, 013001 (2017).

3. J. J. Gil and R. Ossikovski, Polarized Light and the Mueller Matrix Approach (CRC Press, 2016).

4. J. J. Gil, Eur. Phys. J. Appl. Phys. 40, 1 (2007).

5. J. C. Petruccelli, N. J. Moore, and M. A. Alonso, Opt. Commun. 283, 4457 (2010).

6. J. M. Auñón and M. Nieto-Vesperinas, Opt. Lett. 38, 58 (2013).

7. O. Gamel and D. F. V. James, J. Opt. Soc. Am. A 31, 1620 (2014).

8. C. J. R. Sheppard, M. Castello, and A. Diaspro, J. Opt. Soc. Am. A 33 1938 (2016)

9. A. Norrman, A. T. Friberg, J. J. Gil, and T. Setälä, J. Eur. Opt. Soc.Rapid Publ. 13, 36 (2017).

10. J. J. Gil, A. Norrman, T. Setälä, and A. T. Friberg, Phys. Rev. A 97, 023838 (2018).

11. A. Norrman, T. Setälä, and A. T. Friberg, J. Opt. Soc. Am. A 28, 391 (2011).

12. A. Norrman, T. Setälä, and A. T. Friberg, Opt. Lett. 40, 5216 (2015).

13. Y. Chen, A. Norrman, S. A. Ponomarenko, and A. T. Friberg, Phys. Rev. A 97, 041801(R) (2018).

14. Y. Chen, A. Norrman, S. A. Ponomarenko, and A. T. Friberg, Opt. Lett. 43, 3429 (2018).

15. R. Dorn, S. Quabis, and G. Leuchs, Phys. Rev. Lett. 91, 233901 (2003).

16. K. Lindfors, A. Priimagi, T. Setälä, A. Shevchenko, A. T. Friberg, and M. Kaivola, Nat. Photonics 1, 228 (2007)

17. M. Fischer, B. Srivathsan, L. Alber, M. Weber, M. Sondermann, and G. Leuchs, Appl. Phys. B 123, 48 (2017).

18. J. J. Gil, A. T. Friberg, T. Setälä, and I. San José, Phys. Rev. A 95 , 053856 (2017).

19. J. J. Gil, A. Norrman, A. T. Friberg, and T. Setälä, Opt. Lett. 43, 4611 (2018).

20. J. Tervo, T. Setälä, and A. T. Friberg, J. Opt. Soc. Am. A 21, 2205 (2004).

21. J. J. Gil, J. M. Correas, P. A. Melero, and C. Ferreira, Monogr. Semin. Mat. Garcia Galdeano 31, 161 (2004).

22. J. J. Gil and I. San José, Opt. Commun. 283, 4430 (2010).

23. I. San José and J. J. Gil, Opt. Commun. 284, 38 (2011).

24. T. Setälä, K. Lindfors, and A. T. Friberg, Opt. Lett. 34, 3394 (2009).

25. E. D. Palik, ed., Handbook of Optical Constants of Solids (Academic, 1998). 